\title{
Giant nonlinear optical properties of bismuth thin films grown by pulsed laser deposition
}

\author{
D. R. Liu, K. S. Wu, M. F. Shih, and M. Y. Chern \\ Department of Physics, National Taiwan University, Taipei, 10617, Taiwan
}

Received April 8, 2002

\begin{abstract}
Thin films of Bi were grown by pulsed laser deposition on glass substrates at room temperature. The thickness and roughness of the films were characterized by grazing-incidence $x$-ray reflectivity, and the complex refractive indices were measured in the range from 1.5 to $4 \mathrm{eV}$ by spectroscopic ellipsometry. We performed $\mathrm{Z}$-scan measurements to study the third-order optical nonlinearity of the films. It was found that the $\mathrm{Bi}$ films exhibited an unusually large nonlinear refractive coefficient, $n_{I} \sim 1.24 \times 10^{-1} \mathrm{~cm}^{2} / \mathrm{kW}$ and nonlinear absorption coefficient, $\alpha_{I} \sim-3.97 \mathrm{~cm} / \mathrm{W}$, at low laser intensity, $\sim 60 \mathrm{~kW} / \mathrm{cm}^{2}$. This anomaly is believed to have an origin related to melting of the Bi films at the focus spot by the laser beam. () 2002 Optical Society of America

OCIS codes: $190.4720,190.4400,190.4870,190.3270,310.6860$.
\end{abstract}

The nonlinear behavior of complex refractive indices is responsible for many interesting optical phenomena. Optoelectronic materials with a large and fast response in their nonlinear refractive indices are useful for the applications of high-speed all-optical switching and modulation. ${ }^{1-3}$ Composite materials containing Bi nanoparticles were known to have large optical nonlinearity. ${ }^{4}$ However, to our knowledge, the nonlinear optical properties of continuous thin Bi films have not yet been studied, so it is interesting to prepare and measure such films.

It has been reported that the optical properties of Bi thin films grown by thermal evaporation depend strongly on the film thickness. ${ }^{5}$ Pulsed laser deposition (PLD) is a thin-film-growth technique known for the capability to produce high-quality oxide films, such as high-temperature superconductors, ferroelectrics, and giant magnetoresistance materials. ${ }^{6}$ It has also been reported that $\mathrm{Bi}$ thin films grown by PLD have better optical properties, independent of the film thickness, because of their smaller surface roughness. ${ }^{7}$ However, PLD has the disadvantage of producing undesired particulates that condense in the films. The film quality can be poor without careful control of experimental parameters. Therefore, the technique of grazing-incidence x-ray reflectivity (GIXR) was employed to examine and control the metrology, i.e., the thickness and roughness, of the films. We optimized the growth conditions according to the results of x-ray reflectivity to obtain highquality $\mathrm{Bi}$ films with small roughness and precise control of film thickness.

The optical properties of the samples were studied by the Z-scan method, originated by Hill et al. ${ }^{8}$ and quantitatively developed by Van Stryland and coworkers, ${ }^{9,10}$ which is one of the most successful techniques for measuring third-order nonlinearity. The method employs a focused intense Gaussian beam to induce a spatially dependent change in the complex refractive index of a nonlinear material. Consequently, an effective lens with modified transmittance is established, causing the beam to refocus or defocus. ${ }^{11}$
Therefore, by movement of the sample back and forth along the optical path ( $z$ axis) about the focal point, the effects of nonlinear refraction and nonlinear absorption can be easily measured by the change in intensity in the far field. In this Letter we report that giant optical nonlinearity, which is $\sim 10^{5}$ times larger than that of composites containing Bi nanoparticles, ${ }^{4}$ was revealed in (continuous) Bi films.

The Bi thin films were grown in vacuum on Corning glass substrates at room temperature, above which a layer of $\mathrm{Al}_{2} \mathrm{O}_{3}$ was subsequently grown, also by PLD, to prevent oxidation of the $\mathrm{Bi}$. The films were deposited by a $\mathrm{KrF}$ (248-nm-wavelength, 23-ns pulse-length) excimer laser with an energy of $140 \mathrm{~mJ} /$ pulse and a fluence of $1.40 \mathrm{~J} / \mathrm{cm}^{2}$ at the $\mathrm{Bi}$ target. We equipped the laser with an energy monitor to stabilize the pulse energy so that the deposition rate was kept at $\sim 0.1 \mathrm{~nm} /$ pulse throughout film growth. Rastering the laser beam on the targets by use of a scanning mirror made the thickness of the film uniform. The deposition chamber was pumped by a turbo molecular pump to yield a base pressure of $5 \times 10^{-8}$ Torr.

After film growth, a high-resolution x-ray diffractometer equipped with a parabolic Göbel mirror and a monochromating channel-cut crystal was used for measurements of glancing-angle x-ray powder diffraction (GAXRD) and GIXR. For GAXRD, fixing the incidence angle of the parallel x-ray beam at $5^{\circ}$ with the sample surface maximized the signal-to-noise ratio, while the detector (2-theta) was scanned from $10^{\circ}$ to $90^{\circ}$. Figure 1 shows the result, in which sharp diffraction peaks from $\mathrm{Bi}$ were observed, indicating the high crystalline quality of the Bi layer. However, the diffraction peaks associated with $\mathrm{Al}_{2} \mathrm{O}_{3}$ were absent, indicating that the phase is probably amorphous when the film is grown at room temperature. For GIXR, the copper $\mathrm{K}_{\alpha 1}$ x-ray reflectivity of the $\mathrm{Al}_{2} \mathrm{O}_{3}-\mathrm{Bi}$ multilayer film was measured up to $3^{\circ}$ in 2-theta, as shown in Fig. 2. A simulation of the reflectivity curve by the program REFS, ${ }^{12}$ also shown in Fig. 2, indicates that the thicknesses of $\mathrm{Al}_{2} \mathrm{O}_{3}$ and $\mathrm{Bi}$ are 


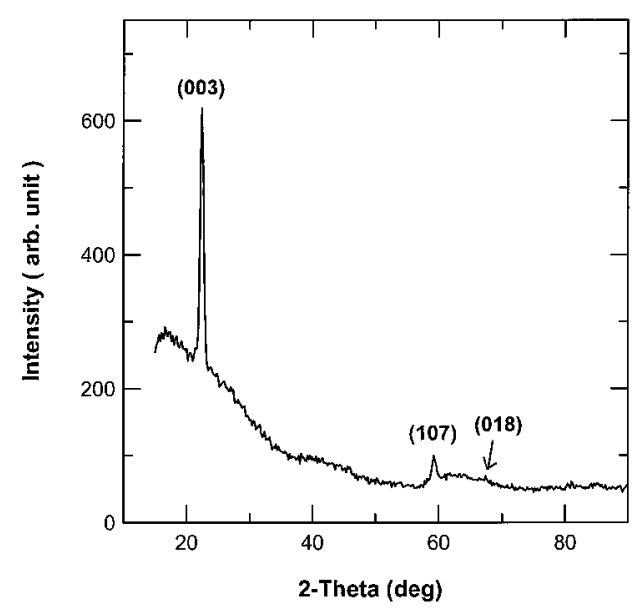

Fig. 1. GAXRD pattern of an $\mathrm{Al}_{2} \mathrm{O}_{3}-\mathrm{Bi}$ multilayer film.

estimated to be 16.1 and $7.0 \mathrm{~nm}$, respectively, and the $\mathrm{rms}$ roughness of the surface and the interfaces of the film is $\sim 0.8 \mathrm{~nm}$.

The (linear) refractive index $n$ and the extinction coefficient $k$ of the films were measured with a rotating-polarizer spectroscopic ellipsometer ranging from 1.5 to $4 \mathrm{eV}$. To derive $n$ and $k$ of the Bi layer sandwiched between the $\mathrm{Al}_{2} \mathrm{O}_{3}$ top layer and the glass substrate, we first determined the complex refractive index of a single $\mathrm{Al}_{2} \mathrm{O}_{3}$ layer of known thickness grown under the same conditions by the rotating-polarizer spectroscopic ellipsometer as a reference. Since there was no peak found in the ellipsometric parameters, $\tan \Psi$ and $\cos \Delta$, in the spectrum, we used the smooth Cauchy functions $n=A+B / \lambda^{2}+C / \lambda^{4}$ and $k=D / \lambda+E / \lambda^{3}+F / \lambda^{5}$ to fit the data for the $\mathrm{Al}_{2} \mathrm{O}_{3}$ layer. The fitting results $\left(A=1.712, B=-3.09 \times 10^{-3}, C=5.71 \times 10^{-4}\right.$, $D=0.015, E=-1.41 \times 10^{-3}, F=1.49 \times 10^{-4}$ ) are similar to the values from the literature. ${ }^{13}$ Then, we used the four-phase model (air- $\mathrm{Al}_{2} \mathrm{O}_{3}-\mathrm{Bi}$-glass) to directly derive $n$ and $k$ of the (sandwiched) Bi layer from the ellipsometric data. The spectra of $n, k$, and the absorption coefficient, $\alpha(=4 \pi k / \lambda)$, shown in Fig. 3, are consistent with those of single-layer Bi films grown by PLD that were described in the literature. $^{7}$

For Z-scan measurements with a setup similar to that described in the literature, ${ }^{9,10}$ the light source was a cw, diode-pumped, frequency-doubled $\mathrm{Nd}: \mathrm{VO}_{4}$ laser $(\lambda=532 \mathrm{~nm})$. We performed the measurements by focusing a nearly ideal Gaussian laser beam with a focal length of $\sim 5 \mathrm{~cm}$ and $\sim 2$-mm diameter onto the thin-film samples. The minimal beam waist at the focal spot was estimated to be $15 \mu \mathrm{m}$, where the laser intensity was $\sim 60 \mathrm{~kW} / \mathrm{cm}^{2}$ for a power of $100 \mathrm{~mW}$. We used an electronic shutter to generate a train of laser pulses (pulse duration, $\sim 25 \mathrm{~ms}$ ) to avoid burning the films. To determine the coefficients for nonlinear refraction and nonlinear absorption, we used two sizes of apertures, a small aperture $(S=0.083)$ and an open aperture $(S=1)$, respectively, in the far field for the intensity measurements. (Here, $S$ is the linear transmittance of an aperture,${ }^{10}$ i.e., the ratio of the power passing through the aperture to the total power of the laser beam.)

Figures 4(a) and 4(b) show the results of the Z-scan measurements with small and open apertures, respectively. The most striking observation is that the apparently large nonlinear effect, $\sim 10 \%$, occurred with only a relatively weak incident intensity, $60 \mathrm{~kW} / \mathrm{cm}^{2}$. We analyzed the data further with theoretical procedures described in the literature to obtain quantitatively the nonlinear refractive coefficient, $n_{I}$, and the nonlinear absorption coefficient, $\alpha_{I}{ }^{10,14}$ The theoretical fitting curve, shown in Fig. 4(c), yields the result that $n_{I}$ and $\alpha_{I}$ are $1.24 \times 10^{-1} \mathrm{~cm}^{2} / \mathrm{kW}$ and $-3.97 \mathrm{~cm} / \mathrm{W}$, respectively. Or, equivalently, the (average) changes in the refractive index $\Delta n$ and the absorption coefficient $\Delta \alpha$ of the Bi films are as large as 7.4 and $-2.4 \times 10^{5} \mathrm{~cm}^{-1}$, respectively. Compared with the films containing Bi nanoparticles, $n_{I}$ is $\sim 10^{5}$ times larger, and the required intensity is $\sim 60,000$ times weaker. ${ }^{4}$

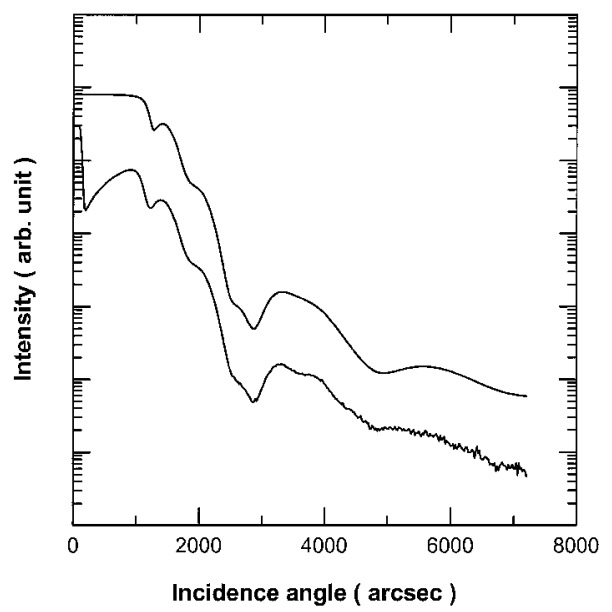

Fig. 2. Experimental and simulated GIXR profiles of an $\mathrm{Al}_{2} \mathrm{O}_{3}-\mathrm{Bi}$ multilayer film. The simulated curve is shifted up by 1 order of magnitude for comparison.

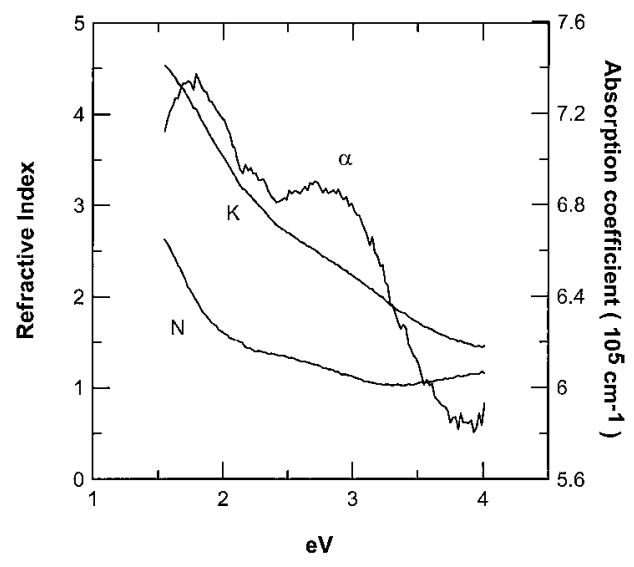

Fig. 3. Spectra for the real part, $n$, and the imaginary part, $k$, of the complex refractive index and the absorption coefficient, $\alpha$, for the $\mathrm{Bi}$ layer in an $\mathrm{Al}_{2} \mathrm{O}_{3}-\mathrm{Bi}$ multilayer sample. 

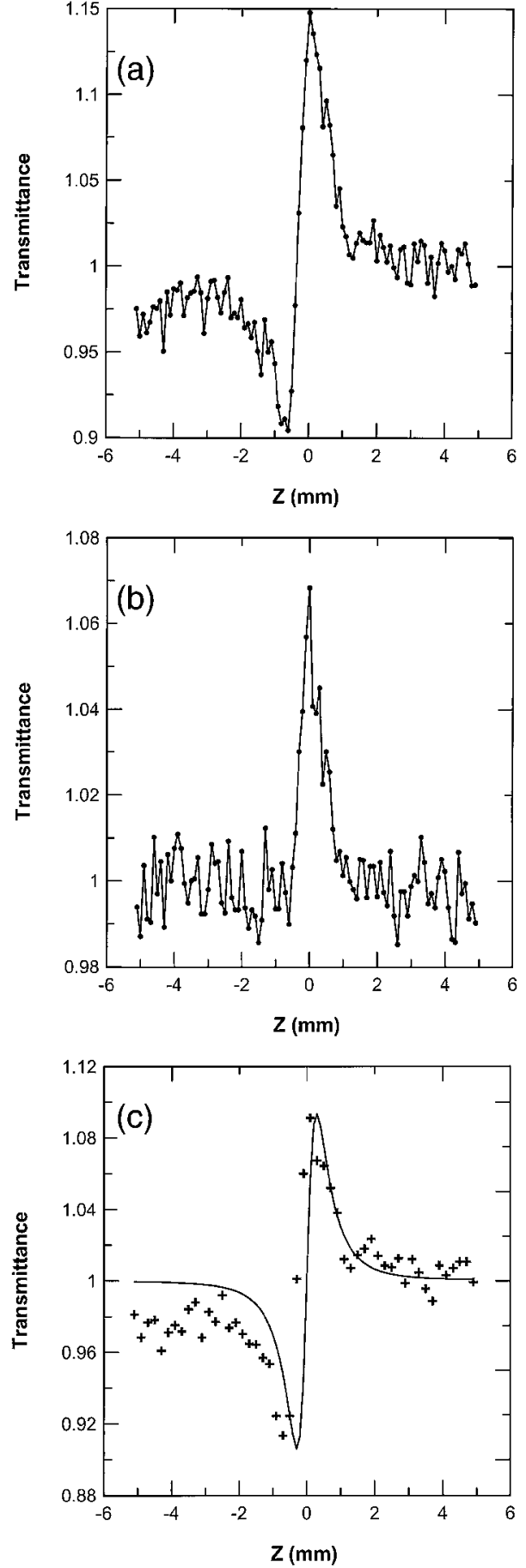

Fig. 4. (a) z-scan transmittance of an $\mathrm{Al}_{2} \mathrm{O}_{3}-\mathrm{Bi}$ multilayer film with a small aperture $(S=0.083)$. (b) z-scan transmittance with an open aperture $(S=1)$. (c) Normalized result (crosses) for the data in (a) divided by those in (b), and the theoretical fitting curve (solid curve) with $\alpha_{I}=-3.97 \mathrm{~cm} / \mathrm{W}$ and $n_{I}=1.24 \times 10^{-1} \mathrm{~cm}^{2} / \mathrm{kW}$.

The complex refractive indices were also measured by spectroscopic ellipsometry at elevated temperatures up to $271^{\circ} \mathrm{C}$, the melting point of $\mathrm{Bi}$. The temperature coefficients, $\mathrm{d} n / \mathrm{d} T$ and $\mathrm{d} \alpha / \mathrm{d} T$, at $532 \mathrm{~nm}$ are of the order of $10^{-4} / \mathrm{K}$ and $10^{0} / \mathrm{cm} \mathrm{K}$, respectively. These numbers are so small that, even with a steep temperature gradient, the so-called thermal-lensing effect cannot account for the large nonlinear effects that were observed. Although the hypothesis still needs to be verified, the giant nonlinearity of the $\mathrm{Bi}$ thin films might be explained with the formation of liquid particles of $\mathrm{Bi}$. Since, among metals, the thermal conductivity, $0.079 \mathrm{~W} / \mathrm{cm}^{\circ} \mathrm{C}$, of $\mathrm{Bi}$ is relatively poor and the melting point is relatively low, it is expected that the focused laser beam provides sufficient energy to melt the $\mathrm{Bi}$ film locally, as its thickness is only $\sim 7.0 \mathrm{~nm}$. A simplified calculation, considering only the heat loss from the top and bottom surfaces, shows that it takes only $\sim 20 \mathrm{~ns}$ for the $100-\mathrm{mW}$ laser to melt the $\mathrm{Bi}$ contained in a $15-\mu \mathrm{m}$-diameter thin disk. On melting, the continuous Bi film breaks up to form small liquid particles because of the cohesive force. Because of the convex shape of the liquid particles, the wave front of the laser beam could be altered so the variation in far-field intensity as a function of $z$ could be observed. Studies of how to improve and understand the nonlinear optical properties of $\mathrm{Bi}$ and related materials are under way.

This work was supported by the National Science Council and the Ministry of Education of the Republic of China. M.-Y. Chern's e-mail address is mychern@ccms.ntu.edu.tw.

\section{References}

1. G. I. Stegeman and R. H. Stolen, J. Opt. Soc. Am. B 6, 652 (1988).

2. P. W. E. Smith, Proc. SPIE 1852, 2 (1993).

3. V. Mizrahi, K. W. DeLong, G. I. Stegeman, M. A. Saifi, and M. J. Andrejco, Opt. Lett. 14, 1140 (1989).

4. Z. Pan, S. H. Morgan, D. O. Henderson, S. Y. Park, R. A. Weeks, R. H. Magruder III, and R. A. Zuhr, Opt. Mater. 4, 675 (1995).

5. R. Atkinson and P. H. Lissberger, Thin Solid Films 17, 207 (1973).

6. D. Chrisey and G. Hubler, eds., Pulsed Laser Deposition of Thin Films (Wiley-Interscience, New York, 1994), and references therein.

7. J. C. G. de Sande, T. Missana, and C. N. Afonso, J. Appl. Phys. 80, 7023 (1996).

8. J. Hill, G. Parry, and A. Miller, Opt. Commun. 43, 151 (1982).

9. M. Sheik-Bahae, A. A. Said, and E. W. Van Stryland, Opt. Lett. 14, 955 (1989).

10. M. Sheik-Bahae, A. A. Said, T. H. Wei, D. J. Hagan, and E. W. Van Stryland, IEEE J. Quantum Electron. 26, 760 (1990).

11. D. A. B. Miller, M. H. Mozolowski, A. Miller, and S. D. Smith, Opt. Commun. 27, 133 (1978).

12. D. K. Bowen and B. K. Tanner, Nanotechnology 4, 175 (1993).

13. R. Sema, J. C. G. de Sande, J. M. Ballesteros, and C. N. Afonso, J. Appl. Phys. 84, 4509 (1998).

14. P. B. Chapple, J. Staromlynska, J. A. Hermann, T. J. McKay, and R. G. McDuff, J. Nonlinear Opt. Phys. Mater. 6, 251 (1997). 